\title{
Review Paper: Burn Coverage Technologies: Current Concepts and Future Directions
}

\author{
ClifFord PEREIRA, ${ }^{1, *}$ WARREN GOLD ${ }^{2}$ AND DAVID HERNDON ${ }^{2}$ \\ ${ }^{1}$ Department of Surgery, Harbor UCLA Medical Center \\ 1000 W Carson Street, Torrance, Los Angeles, California, USA 90502 \\ ${ }^{2}$ Department of Surgery, Shriners Hospital for Children \\ 815 Market Street, Galveston, Texas, USA 77550
}

\section{INTRODUCTION}

\begin{abstract}
Q kin serves as a protective barrier against the environment. Loss of integrity of the skin through burn injuries can lead to major disability and even death [1]. The accomplishments of the past decade have placed us in the midst of an exciting paradigm shift from what used to be of primary concern (i.e., mortality) to areas that focus on improving the quality of life of burn survivors. Impaired healing and excessive scarring are a major source of morbidity for many burn survivors [2]. The primary goal of treatment of burn wounds are the rapid wound closure and a functional and aesthetically satisfactory scar [2].

Anatomically and functionally the skin has two layers; a superficial layer - the epidermis that provides a barrier against infection and moisture loss; and a deeper dermal layer that is responsible for the elasticity and mechanical integrity of the skin. The epidermal layer is a stratified layer mainly composed of keratinocytes, with proliferating basal cells at the innermost layer and the keratinized, relatively
\end{abstract}

\footnotetext{
*Author to whom correspondence should be addressed. E-mail: cpereira72@yahoo.com
} 
impermeable outer stratum corneum layer at the surface. Other cells of the epidermis include Langerhans cells, which function in immune regulation, and melanocytes that produce skin pigment. The dermis consists of fibroblasts, endothelial cells, smooth muscle cells, and mast cells interspersed within extracellular matrix which forms the bulk of the dermis.

Cutaneous wound healing is a dynamic process involving blood cells, parenchymal cells, extracellular matrix, and growth factors and occurs in three overlapping phases i.e., inflammation, re-epithelialization, and remodeling [3]. Depending upon the intensity of heat and the time the skin is exposed to this heat, burn injuries can vary in thickness from superficial to partial thickness to full thickness burns. Superficial burns involve the epidermis and heal rapidly from epidermal remnants in the wound and surrounding skin. Partial thickness wounds involve the superficial portions of the dermis and healing of these wounds depends upon the regeneration of epidermal remnants from sweat and sebaceous glands found in the undamaged deep dermis. Both the epidermis and dermis are completely destroyed in full thickness wounds, healing of these wounds depend upon wound contracture and re-epithelialization from surrounding undamaged skin. If the burn wounds involve a large surface area of the body, wound coverage based upon the body's natural healing processes is overwhelmed and requires surgical intervention in terms of temporary coverage with dressings, or permanent coverage with skin grafts.

Burn wound management has changed in recent decades, with early tangential excision and wound closure being the gold standard [4]. It has been clearly demonstrated that early wound closure leads to reduction in the post-burn hypermetabolic state in the acute phases of burn injury and improved epidermal-mesenchymal communication with rapid wound closure, reduces the frequency of developing fibrotic conditions, such as scar hypertrophy and contractures [5]. Autologous skin grafts however have limited availability and are associated with additional scarring. Severe burn patients invariably lack sufficient adequate skin donor sites. Additionally, skin grafting creates additional donor site wounds equivalent to second degree burns thus further increasing the total body surface area affected [6].

These difficulties have stimulated the development of and use of skin substitutes and replacement materials of natural and biotechnological origin. Ideally, skin substitutes for wound healing should replace all the structures and functions of native skin. Unfortunately, there are currently no engineered skin substitutes that can completely duplicate the complexity of human skin. Available skin substitutes can act as 
temporary wound covers or permanent skin replacements, depending upon their design. Temporarily, they cover the wound thus preventing dehydration and keep the wound bed moist and ready for skin grafting, as and when donor sites heal adequately for reharvesting. Permanent skin substitutes tend to replace one or both layers of skin facilitating wound healing in several different clinical settings. In addition, some skin substitutes stimulate the host to produce a variety of cytokines and growth factors that promote wound healing [6].

This article reviews the current advances in skin replacement technology with a focus on artificial skin constructs rather than naturally available coverage, such as amnion and cadaveric skin and xenografts. The authors have classified skin substitutes as epidermal replacements, dermal replacements and composite (both epidermal and dermal) replacements (see Table 1).

\section{EPIDERMAL RECONSTRUCTION}

\section{Keratinocyte Cultures}

The clonal growth of keratinocytes has been possible for over 20 years [7]. Cell therapy is an emerging therapeutic strategy aimed at replacing or repairing severely damaged tissues with cultured cells. Epidermal regeneration obtained with autologous cultured keratinocytes (cultured epithelial autografts (CEAs) can be life-saving for patients suffering from massive full thickness burns [8,9]. Cultured epithelia avoid the mesh aspect obtained with a split thickness autograft as well as the discomfort of skin graft harvesting for the patient; however there is a time delay of 2-5 weeks for culture of the autologous sheets of keratinocytes. An automated membrane bioreactor was developed in 1999 to produce on a large scale cultured skin grafts at significantly reduced cost and time of transplantation down to two weeks time [10]. The computer uses the obtained information to control medium change and to predict the end of the cultivation largely eliminating the risk of human error. The computer controlled reactor is modular, allowing the production of up to $0.5 \mathrm{~m}^{2}$ of keratinocyte culture sheets at one time [10]. Another advantage of cultured keratinocyte allografts is the large surface area obtained from a relative small biopsy of healthy skin from the patient. However fragility and difficult handling of the grafts, and unpredictable take and extremely high costs are other major disadvantages [8]. Storage and preservation of viable sheets have also been a major problem. Enzymatic detachment of the confluent multi-layered keratinocyte sheet from the irradiated fibroblast feeder 
Table 1. Skin substitutes.

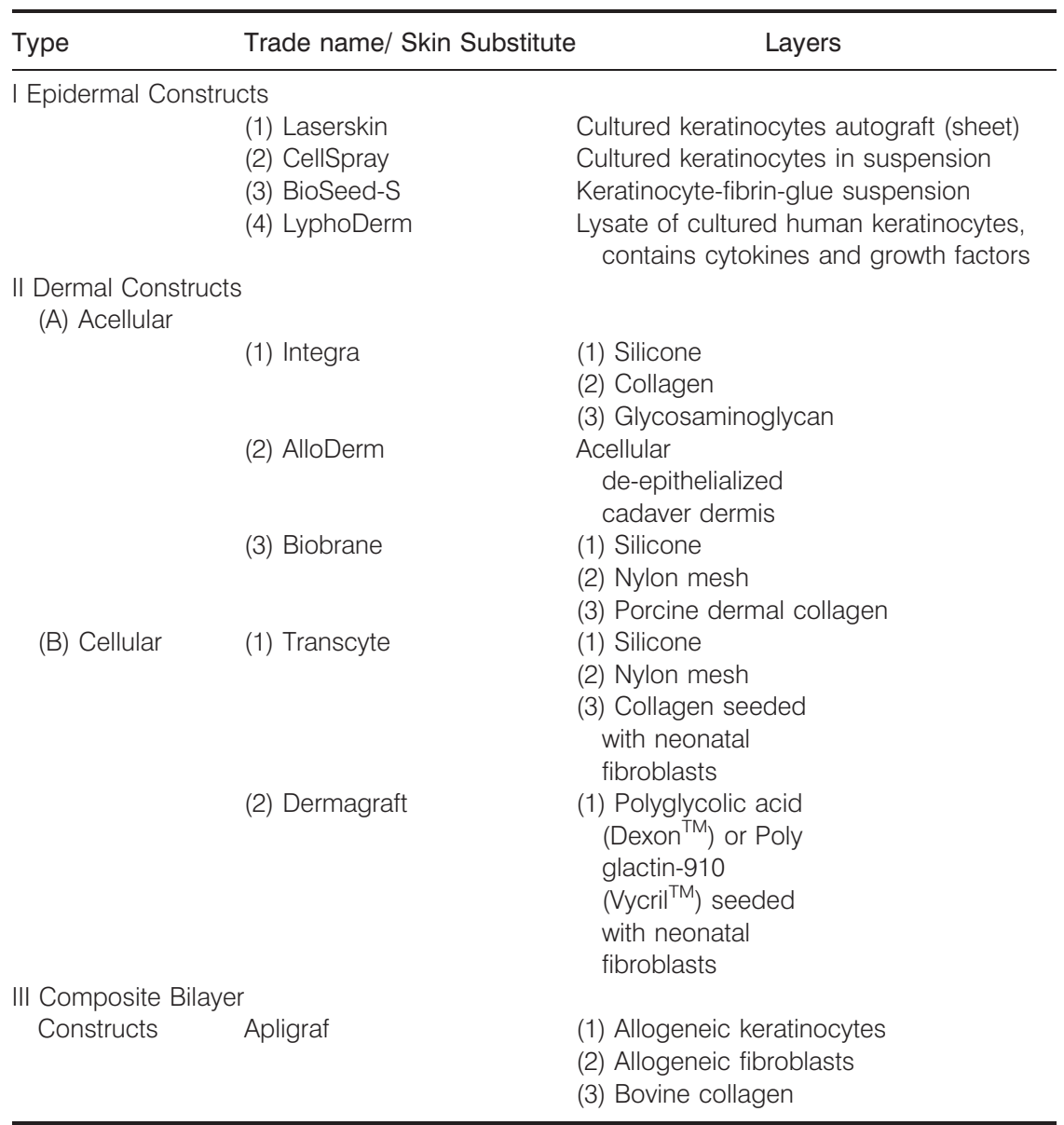

layer which is a critical step of the classable culture method is another factor affecting success of the technique and may be largely responsible for the unpredictable clinical graft take and the fragility of the resultant skin. This critical step of the technique leads to a temporary loss of a634-integrins essential for cellular adhesion [11]. The resulting epithelium is unstable giving rise to spontaneous blistering many months after grafting [12].

Many groups are working on the development of alternative systems for the delivery of cultured autologous keratinocytes, in the hope that this may reduce costs and improve the take and quality of the resulting epidermis. These systems have varied from fibrin-glue 
suspension, fibrin glue sheets, bio-scaffolds, and sprayed cell suspensions. Recently, membranes based on blends of poly(ether) imide (PEI) with polybenzimidazole (PBI) could provide a tissue compatible scaffold with lowered adhesive properties, and might be a useful tool for the transfer of cells by passing enzymatic detachment [13]. CEAs are unsuitable as permanent skin substitute in burn patients with major full thickness burns because they lack the essential dermal component. Dermal substitution with CEAs is required to enhance results [13]. Chimeric xenogeneic-syngeneic graftable sheets consisting of histologically well-organized epidermis presenting basal and suprabasal cell layers previously obtained in vitro have also been developed and tested experimentally with good graft take [14]. Cell phenotyping after healing revealed the presence of only syngeneic keratinocytes, whereas xenogeneic cells were passively eliminated without a total rejection of the chimeric implant. This selective and passive elimination of xenogeneic keratinocytes proceeds through cellular and humoral immunity activation. Data suggest that this chimeric culture method can be used for cutaneous therapies for extensively burned skin [14]. Furthermore, since the ultimate aim in allogeneic and xenogeneic transplantation is to achieve an immunological acceptance and tolerance to these foreign tissues, the chimeric culture approach may provide ways to lighten tolerance phenomena on cutaneous tissues [14]. To circumvent the enzymatic step during the standard cultivation procedure and to simplify handling, keratinocyte cultivation is at present combined with various natural or synthetic carrier materials like polyurethane membrane, siliconecollagen sponges, and fibrin glue [13].

Laserskin by FIDIS Advanced Biopolymer, Italy is a biodegradable keratinocyte delivery system, for keratinocyte grafting. It is a thin transparent membrane, which is semi-synthetic derivative of hyaluronic acid, a naturally occurring ECM molecule and a major component of human skin. The hyaluronic acid is completely esterified to give a benzyl derivative. Microholes are drilled with a laser across the membrane. The seeded keratinocytes are found to populate the pores and grow out as colonies both above and beneath the membrane. The membrane can be peeled from the dish without enzymatic digestion and can be sterilized by gamma radiation $[15,16]$. It is proposed that there is a potential advantage in this membrane not requiring inversion and subsequent cell reorientation for application to the wound bed, and that the presence of hyaluronic acid may promote cell migration, proliferation, and angiogenesis $[15,16]$. 


\section{DERMAL RECONSTRUCTION}

Split-skin graft used on full thickness skin loss in burns is a standard treatment, however it leads to scar formation that is often vulnerable and unstable [2]. It also frequently leads to severe debilitating contractures due to lack of adequate dermal support. Dermal regeneration templates aim at reconstructing the dermal component of skin so that subsequent split thickness skin grafts (STSGs) are more durable and cosmetically superior. In order for the dermal equivalent to fulfill a useful clinical role in burn therapy it should meet certain requirements. The dermal equivalent should be physically strong and easily handled without damage. The fibroblasts within the collagen matrix should remain viable during remodeling. The dermal equivalent must be stable i.e., contraction and reorganization of the lattice by dermal fibroblasts must be complete and the matrix must be resistant to collagenase hydrolysis which is present in the wound site. Dermal replacements have been classified based upon the absence or presence of cellular components, to acellular and cellular dermal construct, respectively.

\section{Acellular Dermal Constructs}

\section{Integra}

Integra (Johnson and Johnson, Hamburg, Germany; Integra Life Sciences Corporation, NJ, USA), has a bilaminar structure, consisting of cross-linked bovine collagen and glycosaminoglycan, coated on one side with a silicone membrane that provides epidermal function. The pore size has been designed at $70-20 \mu$ in order to allow migration of the patient's own endothelial cells and fibroblasts. Following application to a freshly excised wound, the collagen layer is biointegrated with the wound to form a vascular 'neodermis', a process that takes $\approx 3-6$ weeks. Once this stage has been reached, the silastic layer can be removed and an ultra-thin split-skin graft applied [17]. Integra is indicated as an immediate and temporary coverage for acute wounds [18] as well as for full thickness burned patients and post-burn reconstruction [19]. Even though the texture of healed full thickness wounds with Integra is good, it is not, however as supple as those covered by thick autograft [20]. Full thickness autografts therefore remain a gold standard for burns in special areas, such as deep facial burns. Furthermore, Integra is expensive when compared with cadaveric allograft, the learning curve for the surgical technique is steep with high failure rates initially, and requires a two-stage procedure with minimum interval of 3 weeks, 
which can increase hospital stay. Still Integra has an important role in providing immediate wound coverage with insufficient autograft. Donor sites can be recropped more often, since the autografts required are very thin, and it avoids the risks of cross-infection inherent with allografts. It is available immediately and has a fairly long shelf life [17].

\section{Alloderm}

Cadaver graft can be treated with chemical agents to remove epidermal cellular components, which contribute to antigenicity leaving an immunological inert acellular dermal matrix and intact basement membrane complex. This can then be cryopreserved in a combination of dextran, sucrose, raffinose, and EDTA, followed by freezing, which is designed to minimize the loss of biochemical components of the dermis. This type of acellular, dermal transplant (Alloderm) was developed by Life Cell Corporation, Woodlands, Texas, to act as a template and to regenerate into a viable dermis when grafted into a full thickness burn [21]. The ultra-thin meshed autograft from the patient serves as an epidermal component and is applied simultaneously over the dermal allograft. The chief objective of this approach is to augment the amount of dermis, which can be delivered to the wound site while simultaneously decreasing donor site trauma. Following application to a wound bed, it is repopulated by host cells, revascularized, and incorporated into the tissue. The use of Alloderm for burn wounds have shown that fibroblasts populating the dermal allograft have a normal synthetic phenotype and lack the myofibroblast features seen in granulation tissue. Collagen and elastin in these areas are also found to be normal with uniform distribution of hemidesmosomes and anchoring fibrils leading to superior adherence of cultured keratinocytes to the preserved basement membrane complex of Alloderm. Langerhans cells and melanocytes of epidermis and dendritic cells and endothelial cells of dermis reappear in the dermal allograft, indicating the support offered by the acellular dermal allograft to host cell repopulation [22]. In a multicenter study of 67 patients with full thickness or deep partial thickness burns, alloderm was found to be equivalent to thick split-thickness autografts [23].

\section{Biobrane}

Biobrane (Dow Hickman/Bertek Pharmaceuticals, Sugarland, Texas, USA) consists of a synthetic membrane bound to one surface of a nylon mesh and coated with porcine collagen. The silastic membrane serves as an epidermis. Foreskin fibroblasts proliferate on the nylon mesh, secrete collagen, fibrinonectin, growth factors, glycosaminoglycans, and tenasin. 
Thus the material is considered to have an epidermal (the silicone layer) and dermal (the nylon fabric) analogue. The cellular components of this product are non viable at the time of application to the wound surface. Adherence to the wound is promoted by the chemically bonded collagen [24]. The structure is of controlled porosity to allow drainage of exudates from the wound surface. The indications for its use are clear. It is a temporary wound cover suitable for use on superficial burns and donor sites. It is intended to buy time and provide protection to excised burns. Adherence, fluid collection, and subsequent autograft take are similar to results obtained with cryopreserved allograft, providing that bacterial counts are $<10^{5}$ per gram of tissue [25]. But its use requires caution with the particular concern being that of toxic shock syndrome in the pediatric population associated with staphylococcal infection of the exudates that pools below the Biobrane [26].

Acellular dermal replacements perform well with extremely thin autologous split-skin grafts. However, the results have been inconsistent when combined with CEAs [27,51]. No controlled trials have yet demonstrated any long-term benefits for the use of Integra. Its use dramatically increases costs, both directly for the product and indirectly for the increased hospital time and two-step procedure. It also has an increased risk of infection and requires time-consuming dressing changes [28].

\section{Cultured Dermal Constructs}

An in vivo study of cultured artificial dermal substitutes showed that an artificial dermis containing autologous cultured fibroblasts enhances more the re-epithelialization of a full thickness skin defect when compared to an acellular dermal substitute scaffold [29] stressing the importance of incorporating fibroblasts in any bioengineered construct for skin replacement and healing. Alogeneic cultured dermal substitutes (CDS) (artificial skin) can be prepared by culturing fibroblasts on a specially designed scaffold, such as two-layered spongy matrix of hyaluronic acid and atelo-collagen (Col) [19,30].

\section{Transcyte}

Manufactured by Advanced Tissue Sciences, La Jolla, California, Transcyte has an outer polymer layer that is semipermeable and biocompatible. Neonatal fibroblasts are grown in a nylon matrix for 17 days, during which they produce and deposit type I collagen, fibronectin, and growth factors. The material is then stored at $-20^{\circ} \mathrm{C}$ in specially designed cartridges. The inner collagen-containing layer 
adheres quickly to the wound surface. As nylon is not biodegradable, this material cannot act as a dermal substitute. It is recommended for the use on excised full and partial-thickness burns, as an alternative to cadaver skin for patients with third degree burns and to silver sulfadiazine for patients with second degree burns. But its role is currently evolving to target partial-thickness burns. It adheres rapidly to viable wound surface, stimulating epithelialization [31]. Patients with third degree burns require an additional autografting step. Transcyte produces significantly faster healing and less inflammation than split thickness grafts in second degree burns [32,33].

\section{Dermagraft}

Dermagraft (Advanced Tissue Sciences Inc., CA, USA) is a cryopreserved living dermal structure, manufactured by cultivating neonatal allogeneic fibroblasts on a polymer scaffold (poly(glycolic) acid or poly(glactin)-910, marketed as Dexon or Vicryl, respectively) [34]. The fibroblasts become confluent within the polymer mesh, secreting growth factors and dermal matrix proteins (collagens, tenascin, vitronectin, and glycosaminoglycans), thus creating a living dermal structure [35]. This remains viable and metabolically active after implantation into the wound, despite cryopreservation [36]. Dermagraft facilitates healing by stimulating the ingrowth of fibrovascular tissue from the wound bed and re-epithelialization from the wound edges. It does not close the wound and as such, is marketed for stimulating the healing of chronic lesions, such as diabetic foot ulcers, rather than for closing burn wounds [37]. Although it has not been used extensively for burn wounds, it has been used beneath meshed split-skin grafts on full thickness wounds [38]. This could represent a useful means of replacing lost dermal tissue where thin split-skin grafts would be desirable. Further studies are required to determine any long-term benefits in burn wounds [39].

\section{Cultured Dermal Substitutes (CDS)}

Artificial dermis containing autologous cultured fibroblast has been shown in vivo to enhance re-epithelialization of a full thickness skin defect when compared to an acellular dermal substitute scaffold [29], stressing the importance of incorporating fibroblasts in any bioengineered construct for skin replacement and healing [40]. Allogeneic CDSs can be prepared by culturing fibroblasts on a specially designed scaffold, such as two-layered spongy matrix of hyaluronic acid and atelocollagen [41]. A biodegradable salt-leached porous gelatinous scaffold is also appropriate to seed cultured fibroblasts. After 14 days 
of culturing, the fibroblasts exhibit a good affinity to and proliferation on the gelatin scaffolds without showing any signs of biodegradation [40]. CDS can be cryopreserved and transported to other hospitals in a frozen state retaining its ability to release essential cytokines for wound healing particularly vascular endothelial growth factor (VEGF) [41]. Taking into account the manufacturing cost, coupled with the potency of VEGF release, a two-layered sponge of $\mathrm{HA}$ and $\mathrm{Col}$ with a weight ratio of $5 / 2$ is very promising for commercial application [41]. Decellularization of porcine skin to produce an acellular dermal matrix $(\mathrm{ADM})$ for possible biomedical applications has also been described [42]. Autologous CDS allow quick wound bed preparation that would take a thin split-thickness autologous skin graft and have been shown to prevent severe contractures clinically compared to STSGs alone [42]. The application of autologous CDS is promising for the treatment of extensive burn scar contractures particularly in children [42]. The practical application of $\mathrm{ADM}$ and CDS in the management of burn wounds still needs to be clarified.

\section{COMPOSITE BILAYER CONSTRUCTS}

If the basement membrane is preserved, re-epithelialization is rapid. When basement membrane is damaged, epidermal mesenchymal interactions are dependent on resynthesis and structural conformation of all the basement membrane components. This delays re-epithelialization, which in turn leads to contractures in the long term. Understanding of epidermal-dermal interaction is therefore important in determining how such grafting techniques may be developed. Major advances have been made in the understanding of how cells grow into functional tissue and the signaling mechanisms used to achieve this are being slowly dissected [43]. It has been demonstrated that preservation of the basic epithelial-mesenchymal interactions allows for highly complex ex vivo function of epidermal cells [43]. Fibroblast and keratinocyte interaction modulate the levels of MMP-2 and MMP-9 and their inhibitors produced by these cells. This interaction may be critical for a better healing quality at a late stage of the wound healing process [44]. Development of complete dermal-epidermal skin replacement (composite grafts) will undoubtedly greatly simplify burn management. Skin equivalent (organotypic) cultures generate human skin grafts that shortly after grafting normalize their tissue architecture, basement membrane structure, and barrier function [45]. 
Living bilayered skin construct consisting of human neonatal keratinocytes and fibroblasts in a collagen matrix and laboratorygrown bilayered living skin substitute have already been tested clinically to successfully treat a variety of wounds with documented allogeneic cell survival up to 2.5 years after grafting [46-48]. A major limitation of this application is the delayed vascularization of its relatively thick dermal avascular component, which may lead to graft necrosis [49]. A short series using the same allogenic composite model applied to burns patients with $<20 \%$ total body surface area affected has been recently reported, however with little effective take [47]. It is obvious that further development of this model is needed to overcome the hostile wound bed seen in burn patients. Numerous issues still remain to be resolved before this treatment modality becomes widely accepted and before it becomes applicable to cover extensive burn wounds.

\section{Tissue Tech Autograft System}

Tissue tech autograft system (Fidia Advanced Biopolymers Padua, Italy), incorporates an autologous dermal substitute and an autologous epidermal replacement autograft. Each includes a matrix of a hyaluronic acid ester to promote cellular migration and graft take [50]. As a result of extensive research, innovative biodegradable matrices for cell delivery have been developed based on an ester of the naturally occurring extra cellular matrix molecule hyaluronic acid. With this system both autologous dermis and autologous epidermis can be produced which will be completely integrated when grafted.

\section{Composite Biocompatible Skin Graft (CBSG)}

Laserskin technology has led to the development of the composite biocompatible skin graft (CBSG) also referred to as composite biocompatible epidermal graft (CBEG), a composite laserskin graft (CLSG) consisting of autologous keratinocytes cultivated on a pliable hyaluronate-derived membrane (laserskin) that has been preseeded with autologous/allogenic dermal fibroblasts. The CBSGs are much easier to handle than the conventional cultured epidermal autografts and are good human skin substitutes in terms of durability, biocompatibility, high seeding efficacy for keratinocytes, high graft take rate, and low infection rate [51]. 


\section{Apligraft}

Transplantation of cultured autologous keratinocytes as a single-cell suspension in a fibrin glue matrix combined with allogenic skin grafting is also being investigated. Apligraft (Organogenesis, Canton, MD) is derived by combining a gel of type I bovine collagen with living neonatal allogeneic keratinocytes. This composite is currently the most sophisticated commercially available tissue-engineered product. It is available in a ready-to-use form with a 5 day shelf life. In a multicenter randomized trial, there was no difference in the percentage take, however, Apligraft had superior pigmentation, pliability, and vascularity as compared to split skin grafts [52].

\section{Epicell}

Epicell CEA (Genzyme, Boston, MD) consists of autologous keratinocytes and fibroblasts cultured separately then combined on a collagen-glycosaminoglycan matrix [53]. Though results are encouraging, their use in burns remains to be clinically evaluated.

\section{Endothelialized Reconstructed Skin (ERS)}

A new human endothelialized reconstructed skin (ERS), combining keratinocytes, fibroblasts, and endothelial cells (EC) in a collagen sponge has been recently developed, resulting in early vascularization of the ERS, possibly due to inosculation of capillary-like structure networking with the host capillaries, rather than neovascularization, which is a slower process. These results open exciting possibilities for the clinical application of other organ systems that require a rapid vascularization [49].

\section{FUTURE DIRECTIONS}

Tissue engineering combines the principles of cell biology, engineering, and materials science to develop three-dimensional tissues to replace or restore tissue function. Tissue engineered skin lacks several important functions including those provided by hair-follicles, sebaceous glands, sweat glands, and dendritic cells. As clinicians, the authors' aim is to provide wounded patients with the best quality of skin in the shortest possible time. As scientists, the authors have not yet achieved the ultimate goal of a universal 'off the shelf' skin product. As skin substitutes currently available contain at most only two cell types, 
fibroblasts and keratinocytes, they cannot replace all the functions of native skin. Recent and ongoing studies are addressing the preparation of engineered skin containing additional cell types to increase homology to native human skin and improve functional outcome. For example, endothelial cells are currently being studied to initiate angiogenesis in engineered skin grafts in vitro. This would serve to enhance angiogenesis and shorten the time that grafted cells are deprived of nutrients. Human umbilical vein endothelial cells are readily available but can only be used in allogeneic skin substitutes. For clinical application of autologous endothelialized skin substitutes, use of dermal endothelial cells is optimal, and these cells should be isolated from the same skin biopsy used for preparation of fibroblast and keratinocyte cultures. That said, although this is theoretically possible, technical hurdles still need to be overcome. Endothelial cells are slower to grow in culture than keratinocytes and fibroblasts, which delays preparation of endothelialized constructs [54]. In addition, only a small proportion of human dermal microvascular endothelial cells persist during culture of engineered skin grafts, possibly due to apoptosis of these cells [55].

Another limitation of cultured skin is absent or irregular pigmentation. In normal skin, pigmentation results from proper distribution and function of epidermal melanocytes. These serve to protect skin from UV irradiation and psychologically influence the person's body image and personal identity. In preclinical studies selective cultivation and incorporation of melanocytes into skin substitutes has obtained uniform pigmentation [56]. Future models of skin substitutes will benefit from a more thorough understanding of melanocyte function and interaction with keratinocytes and fibroblasts.

Another exciting possibility for development of engineered skin substitutes involves the addition of genetically modified cells. Genetically modified skin substitutes can hypothetically be used to overcome limitations in anatomy and physiology resulting in skin substitutes with greater homology to native human skin and improved performance. Genetically modified cells have been shown to retain their ability to differentiate into a stratified epidermis [57]. Genetic modifications can be used to ectopically express cytokines not normally expressed in a particular cell type to overcome or compensate for deficiencies of engineered skin or alternatively to overexpress growth factors to enhance wound healing. For example, cultured skin substitutes containing keratinocytes genetically modified to overexpress VEGF, enhanced angiogenesis, and improved wound healing in athymic mice [58,59]. Thus overexpressed VEGF in genetically modified skin constructs can 
compensate for the absence of vascular plexus in the grafted cultured skin. Furthermore, genetically modified cultured skin grafts can act as vehicles for cutaneous gene therapy for correction of genetic disorders both cutaneous and systemic. Proteins secreted by keratinocytes, whether native or introduced by genetic modification can reach detectable levels in the serum after grafting $[60,61]$. Preclinical studies have examined the use of genetically modified keratinocyte grafts for delivery of human growth hormone [62,63]. This has definitive use in burn patients since human growth hormone has been shown to improve lean body mass, bone mineral content, enhance wound healing, and reduce hospital stay in pediatric burn patients $[64,65]$. Further, correcting or modifying a circulating protein via genetically modified keratinocytes is much cheaper than producing recombinant proteins. Proteolytic enzymes in the wound exudates tend to lyse topically applied growth factors. Further, of the active protein that remains on the wound bed after these interactions, the final diffusion into the wound tissue is also predictably low. In a study with topically applied basic fibroblast growth factor (bFGF) and epidermal growth factor (EGF) only 1-9\% of the applied dose reached a depth of $1-3 \mathrm{~mm}$ [66]. PDGF-BB, which is the only growth factor in current clinical use, requires a dose of $100 \mu \mathrm{g} / \mathrm{cm}^{2}$ on alternate days, which is almost 3 times the amount that has been shown to be active in genetic studies [67]. This has led to gene therapy emerging as an important method to deliver growth factors to wounds.

Cationic cholesterol-containing liposomal constructs appear to have a great potential for non-viral gene transfer in the skin. They are synthetically prepared vesicles with a positively charged surface. When mixed with negatively charged DNA they form a loose complex and protect the DNA from degradation. The net positive charge of the complex binds readily to negatively charged cell surfaces. Entry into the cell occurs via endocytosis, fusion, lipid exchange, adsorption, and direct membrane poration [68,69]. After cellular uptake, the released cDNA is taken up by the nucleus [70]. Ribosomes transcribe the nuclear cDNA into mRNA, which is then transported to the rough endoplasmic reticulum where it is translated into protein. Following an uptake of these complexes, DNA is discharged into the cytoplasm, transported into a nucleus, and eventually transcribed and translated into a transgenic protein [71].

As a vector for gene therapy, liposomes have become attractive due to their non-viral composition, stability, and ability to interact with the cell membrane [70]. The flexibility in the design of cationic lipid structures 
and the variety of methods used in the liposomal preparation broaden their scope for the therapeutic use [71]. Further, liposomes can be applied by either topical administration or by direct injection of liposomal gene constructs [70,71].

A potential problem of single gene therapy is that increasing the concentration of a single growth factor may not effect all phases of wound healing. A single growth factor cannot counteract all the deficiencies of a burn wound, nor can it control the vicissitudes and complexities of chronic wound healing. Lynch et al. [67] demonstrated that the combination of PDGF and IGF-I was more effective that either growth factor alone in a partial thickness wound healing model created with the use of a dermatome. Sprugel et al. [72] found that a combination of PDGF and bFGF increased the DNA content of wounds in the rat (implanted wound chamber model) better than any single growth factor. Multiple gene transfections to deliver multiple growth factors at strategic time points of wound healing (known as sequential growth factor therapy) is therefore the next logical step in augmenting wound healing.

The concept of a genetic switch is another exciting development where transgenic expression in target cells can be switched 'on' or 'off' depending on the presence or absence of a stimulator, such as tetracycline [73]. Further developments in matrix components and tissue engineering technology offer promise of a slow-release matrix, allowing for prolonged transgenic expression [74]. Use of the skin as a bioreactor for simultaneous wound healing locally and systemic therapy to reduce hypermetabolism postburn is an yet unchartered territory and work in this area promises to be exciting with its clinical relevance.

With recent technical advances in molecular and cell biology that result in large volumes of data, combined with bioinformatics, a new field focusing on the collection and manipulation of biological data, great advances in biological knowledge and understanding are underway. These advances will give rise to the next generation of medical implants and related therapies. While in the past most implants were mostly structural and inert, future products will be much more biologic in nature, taking advantage of mobilizing the inherent biological power of our bodies. While the complexity of skin may be challenging to recapitulate entirely, new or improved functions can be provided by genetic modifications of the tissue constructs [75]. In future, regenerative medicine will aim to bring about stimulation of regeneration of lost tissue rather than repair (scarring). It is believed that inclusion of the growth factors, and cells responsible for fetal scarless healing, into collagen composite scaffolds would optimize the healing 
environment to give a cosmetically aesthetic and functional skin, to match or even supercede split-skin grafts, the present gold standard.

\section{REFERENCES}

1. Pereira, C.T., Barrow, R..E, Sterns, A., Jeschke, M., Hawkins, H., Rosanblatt, J., Kimbrough, C., Lee, J., Sanford, A. and Herndon, D.N. (2006). Age-dependent Differences in Survival After Severe Burns: A Unicentric Review of 1674 Patients and 179 Autopsies over 15 Years, J. American Coll. Surg., 202: 536-548.

2. Martin, P. (1997). Wound healing-Aiming for Perfect Skin Regeneration, Science, 276: 75-81.

3. Kanzler, M.H., Gorsulowsky, D.C. and Swanson, N.A. (1986). Basic Mechanisms in the Healing Cutaneous Wound, J. Dermatol. Surg. Oncol., 12: $1156-1164$.

4. Heimbach, D.M. (1987). Early Burn Excision and Grafting, Surg. Clin. North Am., 67: 93-107.

5. Sawicki, G., Marcoux, Y., Sarkhosh, K., Tredget, E.E. and Ghahary, A. (2005). Interaction of Keratinocytes and Fibroblasts Modulates the Expression of Matrix Metalloproteinases 2 and 9 and their Inhibitors, Mol. Cell Biochem., 269: 209-216.

6. Atiyeh, B.S., Gunn, S.W. and Hayek, S.N. (2005). State of the Art in Burn Treatment, World J. Surg., 29: 131-148.

7. Rheinwald, J.G. and Green, H. (1975). Serial Cultivation of Strains of Human Epidermal Keratinocytes: The Formation of Keratinizing Colonies from Single Cells, Cell, 6: 331-344.

8. Kaiser, H.W., Stark, G.B. and Kopp, J. (1994) Cultured Autologous Keratinocytes in Fibrin Glue Suspension, Exclusively and Combined with STS-allograft - Preliminary Clinical and Histolocical Report of a New Technique, Burns, 20: 23-29.

9. Pellegrini, G., Ranno, R. and Stracuzzi, G. (1999). The Control of Epidermal Stem Cells in the Treatment of Massive Full Thickness Burns with Autologous Keratinocytes Cultured on Fibrin, Transplantation, 68: 868-879.

10. Prenosil, J.E. and Kino-oka, M. (1999). Computer Controlled Bioreactor for Large Scale Production of Cultured Skin Grafts, Ann. NY Acad. Sci., 875: 386-397.

11. Bannash, H., Fohn, M. and Unterberg, T. (2003). Skin Tissue Engineering, Clin. Plast. Surg., 30: 573-579.

12. Woodley, D.T., Peterson, H.D. and Herzog, S.R. (1988). Burn Wounds Resurfaced by Cultured Epidermal Autografts Show Abnormal Reconstitution of Anchoring Fibrils, JAMA, 259: 2566-2571.

13. Altankov, G., Albrecht, W., Richau, K., Groth, T. and Lendlein, A. (2005). On the Tissue Compatibility of Poly Ether Imide Membranes: An in vitro Study on their Interaction with Human Dermal Fibroblasts and Keratinocytes, J. Biomater. Sci. Polym. Ed., 16: 23-42. 
14. Rouabhia, M. (1996). Permanent Skin Replacement using Chimeric Epithelial Cultured Sheets Comprising Xenogeneic and Syngeneic Keratinocytes, Transplantation, 61: 1290-1300.

15. Myers, S.R., Grady, J. and Soranzo, C. (1997). A Hyaluronic Acid Membrane Delivery System for Cultured Keratinocytes: Clinical Take Rates in the Porcine Kerato-dermal Model, J. Burn Care Rehabil., 18: 214-222.

16. Lam, P.K., Chan, E.S.Y., To, E.W.H., Lau, C.H., Yen, S.C. and King, W.W.K. (1999). Development and Evaluation of a New Composite Laserskin Graft, J. Trauma, 47: 918-922.

17. Burke, J.F., Yannas, I.V., Quinby, W.C., Bondoc, C.C. and Jung, W.K. (1981). Successful Use of a Physiologically Acceptable Artificial Skin in the Treatment of Extensive Burn Injury, Ann. Surg., 194: 413-428.

18. Mis, B., Rolland, E. and Ronfard, V. (2004). Combined Use of a Collagenbased Dermal Substitute and a Fibrin-based Cultured Epithelium: A Step Toward a Total Skin Replacement for Acute Wounds, Burns, 30: 713-719.

19. Oka, H., Suenobu, K., Moriguchi, T., Kubo, K., Matsui, H. and Kuroyanagi, Y. (2004). Clinical Trials with Allogeneic Cultured Dermal Substitutes for the Treatment of Burns and Skin Ulcers (Regenerating Medical Millennium Project of the Ministry of Health, Labor and Welfare), Wound Repair Regen., 12: A12.

20. Klein, M.B., Engrav, L.H., Holmes, J.H., Freidrich, J.B., Costa, B.A. and Honari, S. (2005). Management of Facial Burns with a Collagen/Glycosaminoglycan Skin Substitute-prospective Experience with 12 Consecutive Patients with Large, Deep Facial Burns, Burns, 31: 257-261.

21. Wainwright, D.J. (1995). Use of an Acellular Allograft Dermal Matrix (Alloderm) in the Management of Full Thickness Burns, Burns, 21: 243-248.

22. Wainwright, D., Nag, G. and Call, T. (1994). Normal Histological Features Persist in an Acellular Dermal Transplant Grafted in Full Thickness Burns, FASEB, 9-14.

23. Hansbrough, J.F., Dore, C. and Hansbrough, W.B. (1992). Clinical Trials of a Living Dermal Tissue Replacement Placed Beneath Meshed, Splitthickness Skin Grafts on Excised Burn Wounds, J. Burn Care Rehabil., 13: 519-529.

24. Rodeheaver, G.Y., Hartsell, M.L., Faulkner, B.C., Major, D.A. and Foresman, P.A. (1995). Influence of Biobrane Construction on Adherence, J. Burn Care Rehabil., 16: 321-323.

25. Purdue, G.F., Hunt, J.L., Gillespie, R.W., Hansbrough, J.F., Dominic, W.J., Robson, M.C., Smith, D.J., MacMillan, B.G., Waymac, J.P. and Herndon, D.N. (1987). Biosynthetic Skin Substitute versus Frozen Human Cadaver Allograft for Temporary Coverage of Excised Burn Wounds, J. Trauma, 27: 155-157.

26. Egan, W.C. and Clark, W.R. (1988). Toxic Shock Syndrome in a Burn Victim, Burns, 14: 135-138.

27. Lam, P.K., Chan, E.S., Liew, C.T., Law, C., Yen, S.C. and King, W.W. (2002). Combination of a New Composite Biocompatible Skin Graft on a 
Neoepidermis of Artificial Skin in an Animal Model, ANZ J. Surg., 72: 360-363.

28. Druecke, D., Lamme, E.N., Hermann, S., Pieper, J., May, P.S. and Steinau, H.U. (2004). Modulation of Scar Tissue Formation using Different Dermal Regeneration Templates in the Treatment of Experimental Full Thickness Wounds, Wound Repair Regen., 12: 518-527.

29. Lee, S.B., Kim, Y.H., Chong, M.S., Hong, S.H. and Lee, Y.M. (2005). Study of Gelatin-containing Artificial Skin V: Fabrication of Gelatin Scaffolds using a Salt-leaching Method, Biomaterials, 26: 18961-18968.

30. Middlekoop, E., de Vries, H.J., Ruuls, L., Everts, V., Wildevuur, C.H. and Westerhof, W. (1995). Adherance, Proliferation and Collagen Turnover by Human Fibroblasts Seeded into Different Types of Collagen Sponges, Cell Tissue Res., 280: 447-453.

31. Hansbrough, J. (1997). Dermagraft TC (Trancyte) for Partial-thickness Burns: A Clinical Evaluation, J. Burn Care Rehabil., 18: S25-28.

32. Pape, S.A. and Byrne, P.O. (2000). Safety and Efficacy of Transcyte for the Treatment of Partial-thickness Burns, J. Burn Care Rehabil., 21: 390.

33. Noordenbos, J., Dore, C. and Hansbrogh, J.F. (1999), Safety and Efficacy of TransCyte for the Treatment of Partial-thickness Burns, J. Burn Care Rehabil., 20: 275-281.

34. Hansbrough, J.F., Cooper, M.L. and Chohen, R. (1992). Evaluation of a Biodegradable Matrix Containing Cultured Human Fibroblasts as a Dermal Replacement Beneath Meshed Skin Grafts on Athymic Mice, Surgery, 111: 438-446.

35. Hansbrough, J.F., Morgan, J., Greenleaf, G. and Underwood, J. (1994). Development of a Temporary Living Skin Replacement Composed of Human Neonatal Fibroblasts Cultured in Biobrane, A Synthetic Dressing Material, Surgery, 115: 633-644.

36. Cooper, M.L., Hansbrough, J.F., Speilvogel, R.L., Cohen, R., Bartel, R.L. and Naughton, G. (1991). In vivo Optimization of a Living Dermal Substitute Employing Cultured Human Fibroblasts on a Biodegradable Polyglycolic Acid or Polyglactin Mesh, Biomaterials, 12: 243-248.

37. Naughton, G., Mansbridge, J. and Gentzkow, G. (1997). A Metabolically Active Human Dermal Replacement for the Treatment of Diabetic Foot Ulcers, Artif. Organs, 21: 1203-1210.

38. Allenet, B., Paree, F. and Lebrun, T. (2000). Cost-effectiveness Modeling of Dermagraft for the Treatment of Diabetic Foot Ulcers in the French Context, Diabetes Metab., 26: 125-132.

39. Hansbrough, J.F., Dore, C. and Hansbrough, W.B. (1992). Clinical Trials of a Living Dermal Tissue Replacement Placed Beneath Meshed, Splitthickness Skin Grafts on Excised Burn Wounds, J. Burn Care Rehabil., 13: 519-529.

40. Huang, Y.C., Wang, T.W., Sun, J.S. and Lin, F.H. (2005). Epidermal Morphogenesis in an in-vivo Model using Fibroblasts-embedded Collagen Scaffold, J. Biomed. Sci., 12: 855-867.

41. Kuroyanagi, Y., Kubo, K. and Matsui, H. (2004). Establishment of Banking System for Allogeneic Cultured Dermal Substitute, Artif. Organs, 28: 13-21. 
42. Fujimori, Y., Ueda, K., Omiya Y., Kubo, K., Matsui, H. and Kuroyanagi, Y. (2004). Treatment with Autologous Cultured Dermal Substitutes (CDS) for Burn Scar Contracture in Children, Wound Repair Regen., 12: A11.

43. Mitrani, E., Nadel, G., Hasson, E., Harrari, E. and Shimoni, Y. (2005). Epithelial Mesenchymal Interactions Allow for Epidermal Cells to Display an in vivo like Phenotype in vitro, Differentiation, 73: 79-87.

44. Sawicki, G., Marcoux, Y., Sarkhosh, K., Tredget, E.E. and Ghahary, A. (2005). Interaction of Keratinocytes and Fibroblasts Modulates the Expression of Matrix Metalloproteinases -2 and -9 and their Inhibitors, Mol. Cell Biochem., 269: 209-216.

45. Greenberg, S., Margulis, A. and Garlick, J.A. (2005). In vivo Transplantation of Engineered Human Skin, Methods Mol. Biol., 289: 425-430.

46. Nanchahal, J., Dover, R. and Otto, W.R. (2002). Allogeneic Skin Substitutes Applied to Burns Patients, Burns, 28: 254-257.

47. Badiavas, E.V., Paquette, D., Carson, P. and Falanga, V. (2002). Human Chronic Wounds Treated with Bioengineered Skin: Histologic Evidence of Host-graft Interactions, J. Am. Acad. Dermatol., 46: 524-530.

48. Brem, H., Balledux, J., Sukkarieh, T., Carson, P. and Falanga, V. (2001). Healing of Venous Ulcers of Long Duration with a Bilayered Living Skin Substitute: Results from a General Surgery and Dermatology Department, Dermatol. Surg., 27: 915-919.

49. Tremblay, P.L., Hudon, V., Berthod, F., Germain, L. and Auger, F.A. (2005). Inosculation of Tissue-engineered Capillaries with the Host's Vasculature in a Reconstructed Skin Transplanted on Mice, Am. J. Transplant., 5: 1002-1010.

50. Uccioli, L. (2003). A Clinical Investigation on the Characteristics and Outcomes of Treating Chronic Lower Extremity Wounds using the Tissue Tech Autograft System, Int. J. Low Extrem. Wounds, 2:140-151.

51. Chan, E.S., Lam, P.K., Liew, C.T., Lau, H.C., Yen, R.S. and King, W.W. (2001). A New Technique to Resurface Wounds with Composite Biocompatible Epidermal Graft and Artificial Skin, J. Trauma, 50: $358-362$.

52. Waymack, P., Duff, R.G. and Sabolinski, M. (2000). The Effect of a Tissue Engineered Bilayered Living Skin Analog, Over Meshed Split-thickness Autografts on the Healing of Excised Burn Wounds, The Apligraft Burn Study Group, Burns, 26: 609-619.

53. Phillips, T.J., Bhawan, J., Leigh, I.M., Baum, H.J. and Gilchrest, B.A. (1990). Cultured Epidermal Autografts and Allografts: A Study of Differentiation and Allograft Survival, J. Am. Acad. Dermatol., 23: 189-198.

54. Supp, D.M., Wilson-Landy, K. and Boyce, S.T. (2002). Human Dermal Microvascular Endothelial Cells form Vascular Analogs in Cultured Skin Substitutes after Agrafting to Athymic Mice, FASEB J., 16: 797-804.

55. Sahota, P.S., Burn, J.L., Heaton, M., Freelander, E., Suvurna, S.K., Brown N.J. and Mac Neil, S. (2003). Development of a Reconstructed Human Skin Model for Angiogenesis, Wound Repair Regen., 11: 275-284. 
56. Swope, V.B., Supp, A.P., Cornelius, J.R., Babcock, G.F. and Boyce, S.T. (1997). Regulation of Pigmentation in Cultured Skin Substitutes by Cytometric Sorting of Melanocytes and Keratinocytes, J. Investigative Dermat., 109: 289-295.

57. Morgan, J.R., Barrandon, Y., Green, H. and Mulligan, R.C. (1987). Expression of an Exogenous Growth Hormone Gene in Transplantable Human Epidermal Cells, Science, 237: 1476-1479.

58. Supp, D.M., Supp, A.P., Bell, S.M. and Boyce, S.T. (2000). Enhanced Vascularization of Cultured Skin Substitutes Genetically Modified to Overexpress Vascular Endothelial Growth Factor, J. Invest. Dermatol., 114: $5-13$.

59. Supp, D.M. and Boyce, S.T. (2002). Overexpression of Vascular Endothelial Growth Factor Accelerates Early Vascularization and Improves Healing of Genetically Modified Cultured Skin Substitutes, J. Burn Care Rehabil., 23: $10-20$.

60. Fenjves, E.S., Gordon, D.A. and Pershing, L.K. (1989). Systematic Distribution of Apoliproprotein E Secreted by Grafts of Epidermal Keratinocytes: Implications for Epidermal Function and Gene Therapy, Proc. Natl. Acad. Sci. USA, 86: 8803-8807.

61. Fenjves, E.S., Smith, J. and Zaradic, S. (1994). Systemic Delivery of Secreted Protein by Grafts of Epidermal Keratinocytes: Prospects for Keratinocyte Gene Therapy, Hum. Gene Ther., 5: 1241-1248.

62. Vogt, P.M., Thompson, S. and Andree, C. (1994). Genetically Modified Keratinocytes Transplanted to Wounds Reconstitute the Epidermis, Proc. Natl. Acad. Sci. USA, 91: 9307-9311.

63. Jensen, U.B., Jensen, T.G. and Jensen, P.K. (1994). Gene Transfer into Cultured Human Epidermis and its Transplantation onto Immunodeficient Mice: An Experimental Model for Somatic Gene Therapy, J. Invest. Dermatol., 103: 391-394.

64. Suman, O.E., Thomas, S.J., Wilkins, J.P., Mlcak, R.P. and Herndon, D.N. (2003). Effect of Exogenous Growth Hormone and Exercise on Lean Muscle Mass and Muscle Function in Children with Burns, J. Appl. Physiol., 94: 2273-2281.

65. Przkora, R., Herndon, D.N., Suman, O.E., Jeschke, M.G., Meyer, W.J., Chinkes, D.L., Mlcak, R.P., Huang, T. and Barrow, R.E. (2006). Beneficial Effects of Extended Growth Hormone Treatment after Hospital Discharge in Pediatric Burn Patients, Ann. Surg., 243: 796-801.

66. Robson, M.C. and Smith, P.D. (2001). Topical Use of Growth Factors to Enhance Healing, In: Falanga, V. (ed.), Cutaneous Wound Healing, 1st edn, pp. 379-398, Informa Healthcare, London.

67. Lynch, S.E., Nixon, J.C., Colvin, R.B. and Antoniades, H.N. (1987). Role of Platelet-derived Growth Factor in Wound Healing: Synergistic Effects with Other Growth Factors, Proc. Natl. Acad. Sci. USA, 84(21): 7696-7700.

68. Noguchi, A., Furuno, T., Kawaura, C. and Nakanishi, M. (1998). Membrane Fusion Plays an Important Role in Gene Transfection Mediated by Cationic Liposomes, FEBS, 433(1-2): 169-173. 
69. Miller, C.R., Bondurant, B. and McLean, S.D. (1998). Liposome-cell Interactions in vitro: Effect of Liposome Surface Charge on the Binding and Endocytosis of Conventional and Sterically Stabilized Liposomes, Biochem., 37(37): 12875-12883.

70. Williams, R.S., Johnston, S.A. and Riedy, M. (1991). Introduction of Foreign Genes into Tissues of Living Mice by DNA-coated Microprojectiles, Proc. Natl. Acad. Sci. USA, 88(7): 2726-2730.

71. Felgner, P.L. and Ringold, G.M. (1989). Cationic Liposome-mediated Transfection, Nature, 337(6205): 387-388.

72. Sprugel, K.H., McPherson, J.M., Clowes, A.W. and Ross, R. (1987). Effects of Growth Factors in vivo. I. Cell Ingrowth into Porous Subcutaneous Chambers, Am. J. Pathol., 129(3): 601-13.

73. Gossen, M. and Bujard, H. (1992). Tight Control of Gene Expression in Mammalian Cells by Tetracycline-responsive Promoters, Proc. Natl. Acad. Sci. USA, 89(12): 5547-5551.

74. Chandler, L.A., Gu, D.L. and Ma, C. (2000). Matrix-enabled Gene Transfer for Cutaneous Wound Repair, Wound Repair Regen., 8(6): 473-479.

75. Andreadis, S.T. (2007). Gene-modified Tissue-engineered Skin: The Next Generation of Skin Substitutes, Adv. Biochem. Eng. Biotechnol., 103: 241-274. 